\title{
Umbilical Cord Patch Versus Conventional Primary Closure in Gastroschisis and Omphalocele
}

\author{
Alejandro José Hernández Rivero* \\ Department of Pediatric surgery, Central University of Venezuela, Venezuela \\ *Corresponding author: Alejandro José Hernández Rivero, Central University of Venezuela, Caracas, Venezuela
}

Submission: 泟 February 09, 2017; Published: 毕July 16, 2018

\begin{abstract}
Summary
Gastroschisis and Omphalocele are the most frequent and important birth defects of the abdominal wall in the newborn period. In our country we dispose of few hospitals with the necessary infrastructure to care for these infants. Many of them have to wait valuable time to be operated while the institution where they will be transfered is located, increasing the risk of Abdominal Compartment Syndrome and sepsis.
\end{abstract}

Objective: To compare the surgical outcome between the use of the Umbilical Cord Patch versus the Conventional Primary Closure of the abdominal wall in newborns.

Methods: Twenty patients were equally divided into 2 groups. Group A patients underwent abdominal closure using the Umbilical Cord Patch and Group B patients underwent a Conventional Primary Closure of the abdominal wall.

Results: No statistically significant difference was seen in hospitalization time and complications. where as significant statistical difference was seen in intra-abdominal pressure (IAP), onset of oral and survival, if they showed significant statistical between the two procedures.

Conclusion: The Umbilical Cord Patch seems to offer advantages to those infants with delayed closure of the abdominal wall because the technique creates a mesothelial surface in contact with the intestine, it is an autologous tissue, available for use at birth and easily applied. The study showed greater benefits in terms of lower increse of IAP, early onset of oral fedding, and higher survival rate compared with Conventional Primary Closure.

Keywords: Gastroschisis; Omphalocele; Umbilical cord patch; Conventional primary closure

Abbreviations: GQ: Gastroschisis; SCA: Abdominal Compartment Syndrome; HUC: University Hospital of Caracas

\section{Introduction}

Gastroschisis (GQ) is a congenital defect in the abdominal wall characterized by exposure of intestinal loops that protrude through a defect located to the right of a normal insertion umbilical cord [1]. Omphalocele is a congenital malformation in which there is a defect in the abdominal wall where the intestine is covered by a membrane or sac, from which the umbilical cord arises [2].

The incidence of GQ has increased in the last 30 years. According to the International Statistical Classification of Diseases and Related Health Problems, 10th revision (ICD-10), between 1980 and 1990 it was estimated at 1 per 10,000 live births. Currently it is estimated that it is 3 to 7 per 10,000 live births and with a maternal average age below 20 years [3]. The incidence of Omphalocele is estimated between 1.5 and 3 per 10,000 live births and its association with other anomalies is very high [4].

Many hypotheses have been proposed to explain the formation of the GQ, from faults in the differentiation of the embryonic mesenchyme [5], rupture of the amniotic membrane at the base of the umbilical cord [6], abnormal involution of the right umbilical vein [7], disruption of the right vitelline artery [8]; and the most recently formulated by Stevenson in 2009, which proposes that the GQ is caused by the failure of the yolk sac and duct, as well as the yolk vessels, to initially join the allantois and later to the body stem [9]. The formation of the omphalocele can be the result of an incomplete closure of the lateral folds of the embryo during the fourth week of gestation, secondary to a defect in the development of the mesoderm at the level of the yolk sac, which makes most of the abdominal organs remain outside the embryo inside a transparent sac formed by the amnion, parietal peritoneum and Wharton's jelly [4].

In the GQ and in the ruptured Omphalocele, the intestine is affected by being free in the amniotic cavity. There is wall involvement, characterized by an exaggerated amount of fibrin without evidence of ischemia or necrosis. The muscle tissue is 
invaded by collagen. In very severe cases there are deposits of calcium in the serosa. The histological absence of lesions at the level of the myenteric plexuses and the ganglion cells leads to the conclusion that this abnormal accumulation of collagen and fibrin causes the motility disorders observed in these patients. There is evidence that shows that the time of exposure of the intestine to the amniotic fluid is proportional to the degree of damage. Studies in animals showed that the prenatal correction of the defect reversed the changes produced $[4,10]$.

The prenatal diagnosis of these abdominal wall defects is based on the determination of serum alpha-fetoprotein levels in maternal serum between 16 and 18 weeks of gestation [10,11], as well as the characteristic eco-sonographic findings of both GQ ( herniated loops to the right of the umbilical cord and floating freely in the amniotic fluid without surrounding membrane, umbilical cord inserted in the left margin of the defect); as of Omphalocele (mass in the midline that compromises the insertion of the umbilical cord, absence of anterior muscles of the wall, the central umbilical vein that accompanies the herniated defect) [12-14]. The intestinal diameter compared with the gestational age is a useful tool to deduce the damage of the intestine. A diameter greater than 10 mm between weeks 28 and 32 is highly predictive of intestinal deterioration [15].

Survival of patients with GQ is around $90 \%$, with an overall mortality lower than $8 \%$ due mainly to sepsis, while in the Omphalocele the stillbirth rate is estimated at $42.1 \%$, and the total mortality is finds around $68.4 \%$ [4]

The two methods of repair most used in both pathologies are Primary Closure and Deferred Closure after the placement of a silo [16]. The Primary Closure is the reduction of the intestine eviscerated to the abdominal cavity, with synthesis of the fascia and skin. Deferred closure consists of suturing a bag of silastic (Silo) around the edges of the defect of the abdominal wall and by means of progressive plications of the same, introducing the extraabdominal content to the cavity in order to achieve the closure of the fascia and skin in a second surgical time.

In 2007 Svetliza described the Primary Closure of GQ using the principle of Exit Simile (ex utero intrapartum treatment), in which the intrapartum reduction of the herniated viscera is performed, with very encouraging results with respect to the survival of these newborns $[17,18]$.

The umbilical cord is a structure that joins the embryo or fetus to the placenta and is composed of two arteries and a vein in helical or spiral. Wharton's gelatin is a gelatinous substance that surrounds the cord and has a protective function in relation to the umbilical vessels. It contains prostaglandins and a high level of proliferation and differentiation. There is activity of telomerase responsible for the rapid synthesis of the extracellular matrix components that achieve the production and regeneration of cells [19].

The use of the umbilical cord in the repair of abdominal wall defects is well known since the late twentieth century [20]. In 1974, the first satisfactory repair was reported in a newborn with GQ [21].
Although the umbilical cord is associated with low inflammatory reaction, low incidence of dehiscence of the wall, better healing of the surgical wound and a lower percentage of complications related to increased intra abdominal pressure (IAP); ventral hernia formation is reported $[22,23]$. Some authors modified this technique by reinforcing the umbilical cord patch by covering it with a polypropylene mesh [24]. Ionescu et al. [25] described other advantages of the Umbilical Cord Patch in the treatment of GQ. In this way, a mesothelial surface is created in contact with the small intestine, which decreases the rate of infection, and by unloading the tension that implies the primary closure of the aponeurosis, the risk of increasing the PIA is significantly reduced and with it the development of Abdominal Compartment Syndrome (SCA).

The GQ and the broken Omphalocele have represented different medical-surgical problems derived from the inflammation and thickening of the loops. The protrusion of the intestine and its meso through a hole, at a time when it begins to gain greater content and propulsion capacity, cause a situation of disproportion between the visceral volume and the capacity of the abdomen. Thus, the reduction of inflamed viscera may prevent the primary closure of the abdominal wall. When this is achieved, there is a risk of producing an ACS that compromises renal flow and adequate bowel perfusion. A large percentage of newborns have to wait for valuable time to be able to be operated while the institution where they are going to be received is located. It is precisely during this time when there is a greater inflammatory reaction of the intestine, which hinders the primary closure of the abdominal wall defect, and there is an increased risk of ACS. That is why these newborns can benefit from the use of Umbilical Cord Patch since it is a technique provided with a low percentage of complications and a high survival rate.

The research aims to compare the surgical procedures of the Umbilical Cord Patch versus the Primary Closure of the abdominal wall in patients with GQ and Omphalocele who were admitted to the Neonatal Surgery Unit of the Pediatric Surgery Service of the University Hospital of Caracas, in the period included between 2013 and 2015.

\section{Methods}

With the prior authorization of the Ethics Committee of the institution as well as the signing of the informed consent, a prospective controlled clinical trial was conducted. We included neonates with GQ and Omphalocele born in the University Hospital of Caracas (HUC) or in other hospitals in the country and who were referred. We excluded those neonates with GQ and Omphalocele who underwent abdominal closure in two periods (patients with silo or with vacuum-pack system), who had other severe malformations diagnosed in the prenatal period; and those newborns with GQ treated with the Simil-Exit procedure.

The sample of 20 patients was divided into two groups: Group A, constituted by neonates who underwent operative cures of GQ or Omphalocele using Umbilical Cord Patch; and Group B, formed by neonates who underwent operative cures of GQ or Omphalocele through the conventional Primary Closure of the abdominal wall. 
The surgical technique used in 10 patients corresponding to group A (7 GQ and 3 Omphaloceles) was as follows: Under general anesthesia after antisepsis measurements, gut enterotaxia was performed with subsequent reduction of the organs to the abdominal cavity. Then, in the patients with GQ, we proceeded to the dissection plus resection of the umbilical cord stem elements and to the making of the Wharton Gelatin Patch, maintaining its continuity with the aponeurosis on the left side of the defect (Figure 1). Subsequently, the patch was fixed to the aponeurosis with Vicryl ${ }^{\circ}$ 3/0 in continuous suture, occluding the wall defect (Figure 2). The last step consisted of the skin closure with PDS® $4 / 0$ in continuous suture, after releasing it from the aponeurosis. To the patients with Omphalocele, the guts of the Wharton Gelatin were initially released. In second place, the patch was made according to the size of the aponeurotic defect, maintaining the continuity of the Wharton's gelatin with the aponeurosis located in the upper part of the defect. The next surgical time consisted of fixing the patch to the aponeurosis with Vicryl@ 3/0 in continuous suture, occluding the wall defect (Figure 3). Finally, skin synthesis was performed with PDS® 4/0 in continuous suture, after releasing it from the aponeurosis (Figure 4).

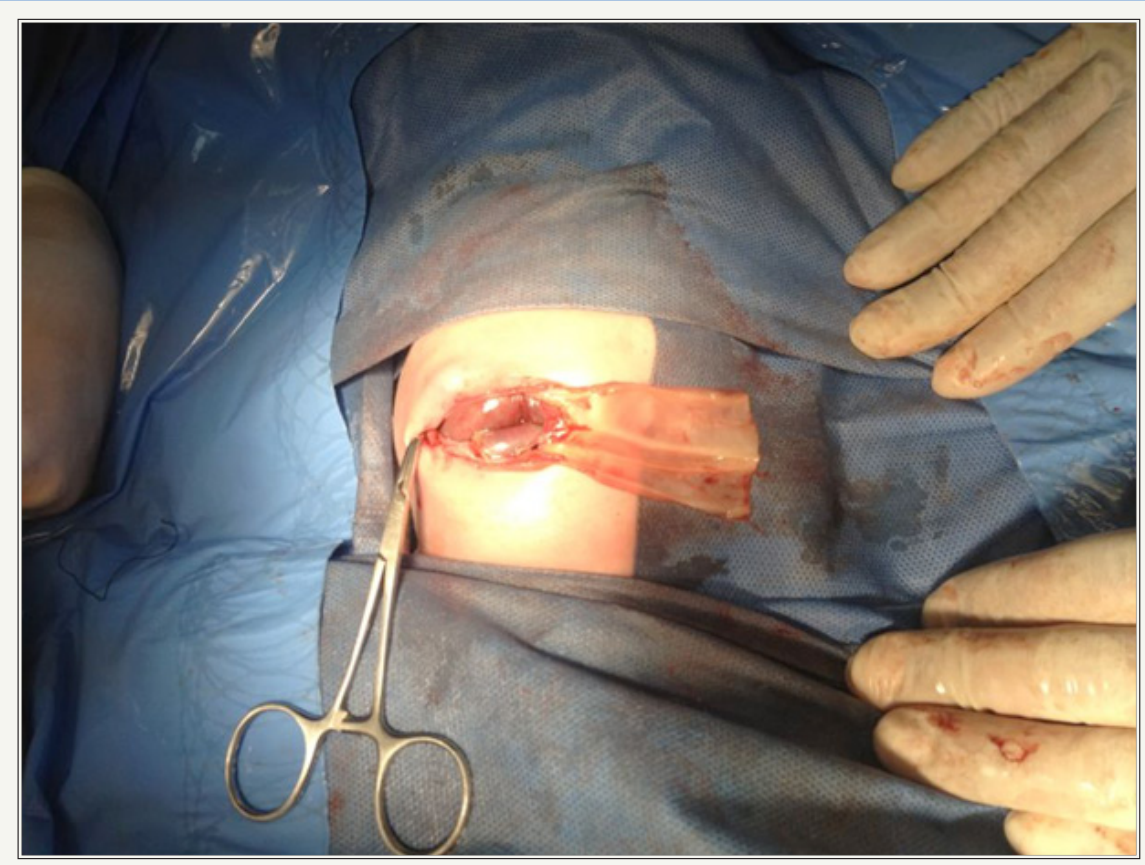

Figure 1: Making of Umbilical Cord Patch (GQ).

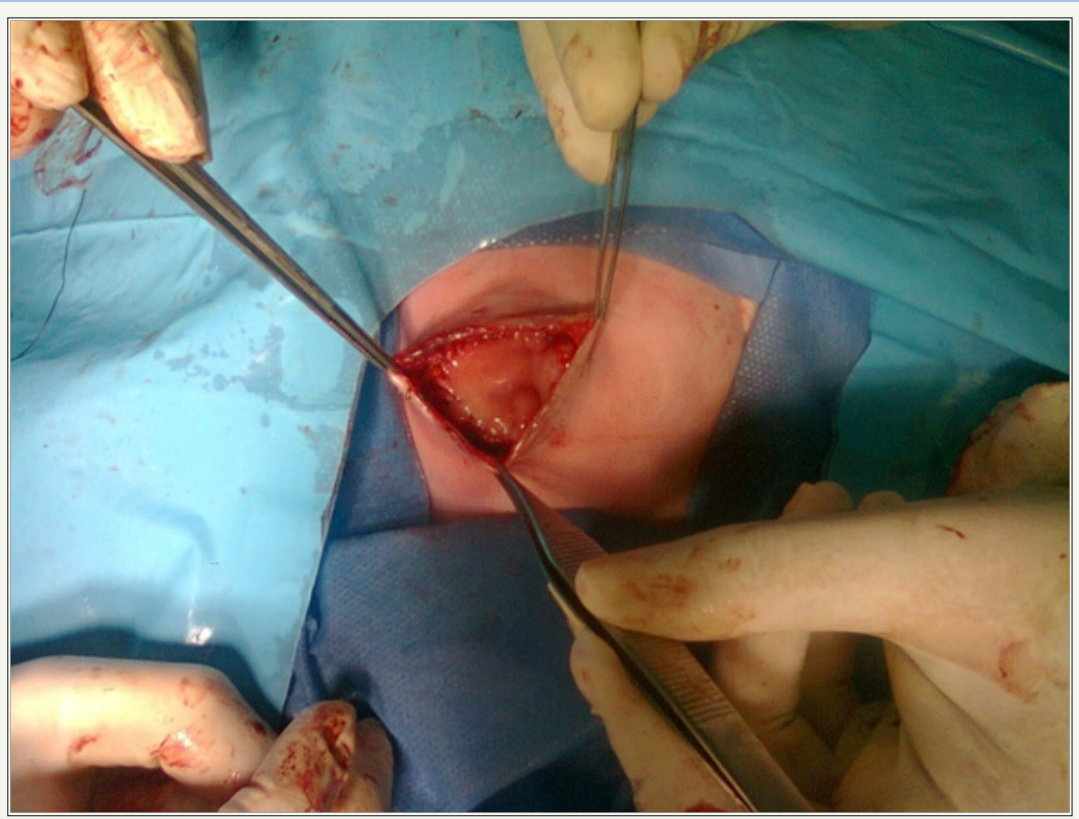

Figure 2: Fixation of the patch to the aponeurosis (GQ). 


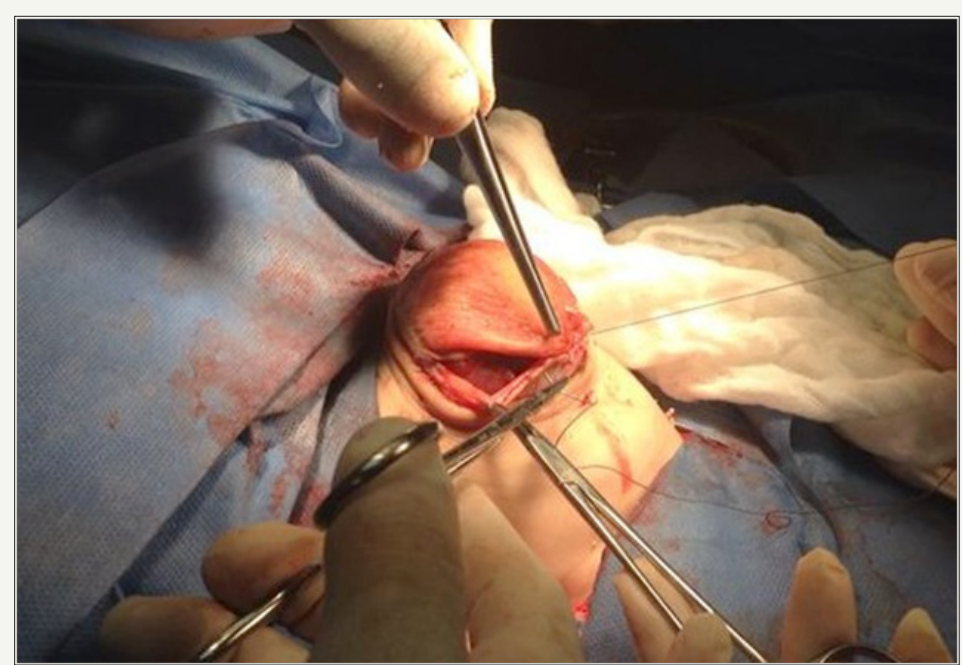

Figure 3: Fixation of the patch to the aponeurosis (Omphalocele).

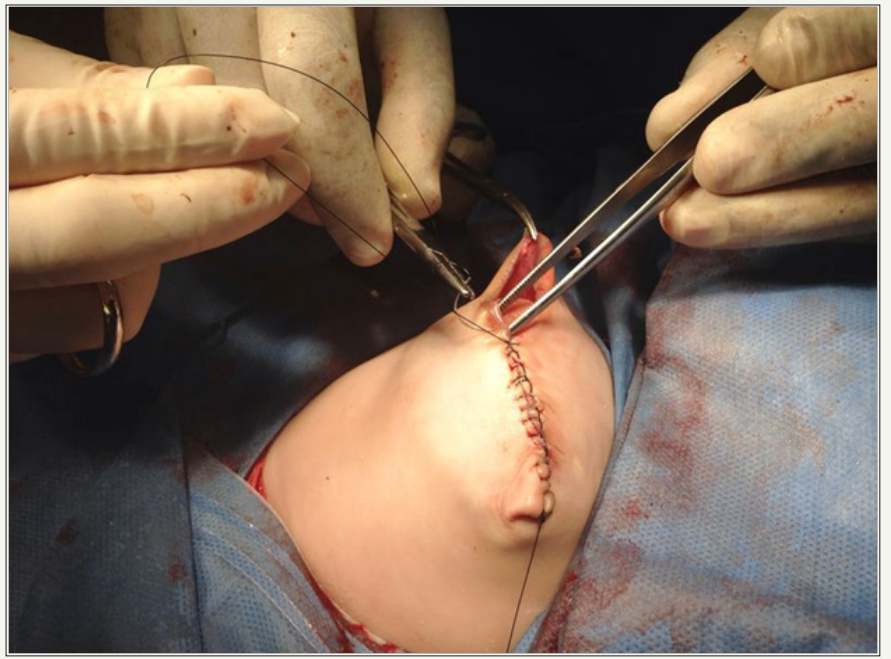

Figure 4: Skin synthesis (Omphalocele).

The other group composed of 10 newborns (7 GQ and 3 Omphaloceles) underwent primary closure of the abdominal wall (Group B). Under general anesthesia after antiseptic measures, gutted enterotaxia was performed with subsequent reduction of the organs to the abdominal cavity and synthesis of aponeurosis with Vicryl@ 3/0 and skin with PDS® 4/0.

All newborns were transferred to the Neonatal Surgery Unit, and once they were stabilized in the servo, the indirect measurement of the IAP was carried out through a bladder catheter. Both groups received intravenous antibiotic therapy and total parenteral nutrition in the postoperative period, with the beginning of tolerance of the oral route when clinical and radiological improvement were evidenced.

The following data were reported: prenatal control, prenatal diagnosis of GQ (in weeks), gestational age (in weeks), patient's lifetime at the time of surgery (in hours), associated malformations, IAP, time of hospitalization, initiation of the oral route, complications and survival.
Calculations were made of average values, standard deviations and percentages in relation to the variables studied. In addition, statistical comparisons were made between both groups; using the $t$ methods of student and modified chi square. A p $<0.05$ was considered significant from the statistical point of view.

\section{Results}

Table 1: Newborns with Gastroschisis or Omphalocele. Variables studied

\begin{tabular}{|c|c|c|c|}
\hline SEXO C.U. & $\begin{array}{c}\text { Parche C.U. } \\
\text { (Group A) }\end{array}$ & $\begin{array}{c}\text { Primary Closure } \\
\text { (Group B) }\end{array}$ & Total \\
\hline Male & 3 & 7 & 10 \\
\hline Female & 7 & 3 & 10 \\
\hline Total & 10 & 10 & 20 \\
\hline
\end{tabular}

Source: Own data. 
We analyzed 20 newborns diagnosed with GQ or Omphalocele who were admitted to the Neonatal Surgery Unit of the University Hospital of Caracas, which were divided into 2 groups (Table 1).

Of the total sample, 14 newborns had a diagnosis of GQ and six presented Omphalocele. Eight of the ten patients in Group A were referred to our center from other hospitals in the Metropolitan area of Caracas, while seven of the ten patients in Group B, similarly, came referrals from other centers.

Variables such as the age of the mothers, gestational age, prenatal control and birth weight showed similar averages in both groups. In relation to the associated malformations diagnosed in the neonatal period, only two patients in Group A were compromised. One presented Right Equine Foot and another was diagnosed with Foramen Oval Permeable. While in Group B, three patients were detected Foramen Oval Permeable and one Functional Stenosis of the Left Branch of the Pulmonary Artery.

Eight of the neonates of Group A merited mechanical ventilation in the immediate postoperative period, while in Group B only seven newborns had an indication for it. The PIA and the beginning of the oral route in the postoperative period showed a statistically significant difference between both groups $(\mathrm{P}<0.05)$. Both the average time for performing the surgical procedure after birth, the duration of surgery, the average time of hospitalization and the percentage of complications did not show a statistically significant difference between both groups. Of the total of 20 newborns in this study, 15 had complications. Eight neonates (80\%) belonged to Group A and seven (70\%) belonged to Group B, there being no difference from the statistical point of view. The complications that the neonates presented in Group A were sepsis of abdominal starting point in two cases, hypertension in two other newborns; a neonate with Omphalocele presented seroma of the surgical wound, another right pneumothorax development after taking a central venous line; in one case clinical evidence of necrotizing enterocolitis was evidenced and in another case the neonate presented clinical signs of neurosifilis. In Group B complications were SCA in two neonates, sepsis of abdominal starting point in two newborns, intestinal perforation in one case; Bilateral pneumonia in a neonate and necrotizing enterocolitis evidenced in a newborn.

Two neonates died in Group A, both with GQ; while six newborns died in Group B, there being a statistically significant difference (P $<0.05$ ) (Table 2).

Table 2: Newborns with Gastroschisis or Omphalocele. Variables studied ${ }^{*}$ ) Statistically significant difference $\mathrm{p}<0.05$.

\begin{tabular}{|c|c|c|}
\hline Variable & Parche C.U. (Group A) & Primary Closure (Group B) \\
\hline Onfalocele & $3 \mathrm{Rn}$ & $3 \mathrm{Rn}$ \\
\hline Gastrosquisis & $7 \mathrm{Rn}$ & $7 \mathrm{Rn}$ \\
\hline Maternal Age & 19.3 years & 19.2 years \\
\hline Gestational age & 36.5 weeks & 35.8 weeks \\
\hline Control Pre-natal & $6 \mathrm{Rn}$ & $5 \mathrm{Rn}$ \\
\hline Birth weight & 2,167 Kgr & 2,275 Kgr \\
\hline Waiting time for Surgery & 67.2 hours & 64.8 hours \\
\hline Surgical Time & 124.8 minutes & 115.4 minutes \\
\hline P.I.A. $\left({ }^{*}\right)$ & $13,5 \mathrm{~cm} \mathrm{H} 2 \mathrm{O}$ & $22,9 \mathrm{~cm} \mathrm{H} 2 \mathrm{O}$ \\
\hline Hospitalization time & 33.43 days & 38.2 days \\
\hline Start VO $(*)$ & 11.13 days & 28.5 days \\
\hline Complicated & 8 & 7 \\
\hline Survival $(*)$ & 8 & 4 \\
\hline
\end{tabular}

Source: Own data: The cause of death of the two newborns of Group A was due to sepsis of abdominal starting point, while of the six neonates who died in Group B, five were similarly due to abdominal starting point sepsis and one due to bilateral pneumonia.

\section{Discussion}

GQ and Omphalocele are the most frequent and important congenital abnormalities of the abdominal wall affecting the newborn, and its incidence, both worldwide and nationally, has increased over the last 20 years. The prognosis of these patients will depend on the degree of intestinal lesion produced in the prenatal period, as well as on the adequate conditions necessary for the post-operative management of these newborns. It is appropriate to affirm of these two malformations, that although it is true, they should be taken to the operating room under the best conditions, the closure of the defect should never be delayed due to the risk of infection and sepsis, as well as the impossibility of bowel reduction. by edema; and the consequent SCA development.

This work aims to evaluate the current utility of the technique described by Samii et al. [21], which consists in making an Umbilical Cord Patch and in this way facilitate the total closure of the abdominal wall defect in a single surgical time; as well as comparing this technique versus Primary Closure of the abdominal wall in newborns with GQ or Omphalocele, in order to provide 
objective evidence on the advantages and disadvantages of each procedure.

In our series there were statistically significant differences between the two groups with respect to the PIA, with the average being much lower in Group A compared to Group B. The fact of achieving the closure of the abdominal wall without tension prevents that all the effects produced by intra-abdominal hypertension such as decreased pulmonary compliance with the consequent pulmonary hypoperfusion and hypoxia; decreased cardiac preload and increased post-load, decreased renal arterial flow and glomerular filtration rate; Hepatic hypoperfusion as well as decreased mesenteric arterial flow which results in ischemia of the intestinal mucosa, local acidosis and bacterial translocation that can lead to septic shock [26]. This seems to be a great advantage of the use of the Umbilical Cord Patch in these newborns in which surgical treatment has been delayed.

Regarding the average hospitalization time, there was no statistically significant difference between the two groups. However, these figures coincide with the vast majority of international literature $[2,4,11]$. A recent study suggests that the hospitalization time of these neonates may be shortened if the gestational age increases [27]. It also concludes that the initiation of the oral route may be earlier in those neonates whose gestational age is above 37 weeks. In our study, neonates from Group A (11.13 days) initiated the oral route earlier compared with Group B (28.5 days), there being a statistically significant difference. This could be a consequence of the fact that the patients who underwent closure by making an umbilical cord patch presented lower IAP figures and therefore the degree of intestinal damage and hypomotility was low in this group. In this way the beginning of the oral precocious route was favored. It should also be noted that the average gestational age of Group A was greater than that of Group B (36.5 weeks versus 35.8 weeks), this result being close to the aforementioned work.

Regarding complications, there was no significant statistical difference between the two groups in relation to the percentage of them. Abdominal starting point sepsis was the most frequent complication in both groups (4 neonates in total), which correlates with the statistics described by other authors [28-30]. Two newborns from Group B developed SCA which represents approximately $13 \%$ of all complications of the 20 patients in our study. This figure correlates with that reported in several literatures $[26,29]$

Of the 8 neonates who died in our study, 7 were due to abdominal starting point sepsis (88\%). The GQ and Omphalocele have classically presented different medical-surgical problems derived from the inflammation and thickening of the intestinal loops that are evidenced in the newborn with these pathologies. When there is a delay in the surgical resolution of these patients, the damage to the intestinal wall is increased, with greater production of fibrin and calcium deposition, conditioning a severe motility disorder, resulting in an increase in the morbidity of the newborn and the development of sepsis [29].
The use of Umbilical Cord Patch in the closure of the abdominal wall in neonates with GQ and Omphalocele seems to offer an advantage for these patients in terms of survival, when compared with the conventional primary closure of aponeurosis and skin. In 1974, Samii and Jafroudi [21] used an umbilical cord patch to close an aponeurotic defect 2 centimeters in diameter in a newborn with GQ. This closure was successful and the patient had as a sequel the formation of a hernia that was corrected years later. Other authors in the 90s and in the first decade of the 21st century used the umbilical cord patch successfully in the repair of GQ with a high survival rate, close to $100 \%$ and fewer complications related to the increase of the PIA; however, the ventral hernia formation did have a high incidence in these neonates [22,31]. Hernández et al [24] reported two cases of neonates with GQ in which they closed the defect with Umbilical Cord Patch, reinforced with a polypropylene mesh attached to the aponeurosis and covered by skin, with good results. In our series, there was evidence of ventral hernia in all infants treated with this technique six months after postoperative control.

Other authors have used the Umbilical Cord Patch for the progressive and phased closure of the aponeurotic defect in newborns with GQ similar to the use of the silo. Werbeck et al. [23] used this technique in 17 neonates with a 100\% survival and a low percentage of infectious complications related to increases in IAP. More recently, Ionescu et al. [25] used the Umbilical Cord Patch for the primary closure of GQ. In their series of 12 neonates, seven were treated with primary closure of the wall, three with silastic silo and two using the umbilical cord patch. These two newborns survived and did not present infectious complications or dehiscence of the suture.

In our study, only two neonates with GQ died who were treated with the Umbilical Cord Patch. The first of these was HIV (+) and VDRL (+) presenting clinical signs of Neurosifilis, and died on the sixth day of age ( $4^{\text {th }}$ postoperative day). The second deceased newborn treated with this technique, occurred at 16 days of age (14 th $^{\text {th }}$ postoperative day) and was due to sepsis secondary to right apical pneumonia. All neonates with Omphalocele who were treated with this procedure survived. Only one of them presented seroma and partial dehiscence of the wound, which closed by second intention.

A high percentage of these neonates are managed by using a silo to achieve staged closure. Recent studies again establish a comparison between Primary Closure and Deferred Closure using silo, with no differences in terms of advantages of a particular technique [32-34]. What is well demonstrated is that infectious complications are responsible for the high morbidity and mortality of these neonates, and that it is related to the delay in the closure of the wall from the moment of birth and the increase in IAP [29].

The use of Umbilical Cord Patch seems to offer advantages to these newborns in which the closure of the abdominal wall has been delayed, since the technique creates a mesothelial surface in contact with the intestine, it is an autologous material, available, easy to Apply; and in our study showed greater advantages in terms 
of the low increase in IAP, earlier start of the oral route and higher percentage of survival compared to conventional primary closure.

\section{References}

1. Briceño L, Calcaño G, Jiménez JO, Gastroschisis (2003) Council of Scientific and Humanistic Development. In: Briceño L, Calcaño G (Eds.), Pediatric surgery ( $1^{\text {st }}$ edn), UCV, Venezuela, pp. 497-516.

2. Tunell WO, Gastroschisis (1995) Nueva Editorial Interamericana. In Ashcraft K, Holder T (Eds.), Pediatric surgery ( $2^{\text {nd }}$ edn), S.A. Mexico, $p p$. 562-572.

3. Kiryluk A, Wieckowska B, Wisniewska K, Czyzewska M, Godula-Stuglik U et al. (2016) Geospatial clustering of gastroschisis in poland: data from the polish registry of congenital malformations (PRCM). Inter J Occup Med Environ Health 29(3): 461-470.

4. Nazer J, Cifuentes L, Aguilar A (2013) Defects of the abdominal wall. Comparative study between omphalocele and gastroschisis. Rev Chil Ped 84(4): 403-408.

5. Duhamel B (1963) Embryology of Exomphalos and Allied Malformations. Arch Dis Child 38(198): 142-147.

6. Shaw A (1975) The myth of gastroschisis. J Pediatr Surg 10(2): 235-244.

7. de Vries PA (1980) The pathogenesis of gastroschisis and omphalocele. J Pediatr Surg 15(3): 245-251.

8. Hoyme HE, Higginbottom MC, Jones KL (1981) The vascular pathogenesis of gastroschisis: intrauterine interruption of the omphalomesenteric artery. J Pediatr 98(2): 228-231.

9. Stevenson RE, Rogers RC, Chandler JC, Gauderer MW, Hunter AG (2009) Escape of the yolk sac: a hypothesis to explain the embryogenesis of gastroschisis. Clin Genet 75(4): 326-333.

10. López J, Castro D, Venegas C (2011) New embryological hypotheses, genetics and epidemiology of Gastroschisis. Bol Med Hosp Infant Mex 68(3): 224-231.

11. Martínez Ferro M (2004) Defects of the abdominal wall. In: Martínez Ferro M, et al. (Eds.), Surgical neonatology, Buenos Aires:Guide Group, Argentina, p. 363-399.

12. Peiró J, Guindos S, Lloret J, Marhuenda C, Torán N, et al. (2005) New surgical strategy in Gastroschisis: simplification of the treatment according to its physiology. Cir Pediatr Barcel 18: 182-87.

13. Forero Caballero H (2002) Congenital defects of the midline of the anterior abdominal wall. In: Forero Caballeo H (Ed.), Neonatal surgical pathology of high risk, Bogotá, Columbia, p. 132-147.

14. Primucci P, Viglanco M, Brignoli V Omphalocele (2009) Rev Arg by Ultrason 8(1): 5-8.

15. Abuhamad A, Mari G, Cortina R, Croitoru D, Evans A (1997) Superior mesenteric artery Doppler velocimetry and ultrasonographic assessment of fetal bowel in gastroschisis: a prospective longitudinal study. Am J Obstet Gynecol 176 (5): 985-990.

16. Villela J, Salinas M, Rodríguez M (2009) Medical-surgical evolution of neonates with gastroschisis according to time, method of abdominal closure and intestinal compromise: six years of experience. Cir Pediatr 22(4): 217-222.
17. Svetliza J, Palermo M, Espinosa A, Gallo M, Calahorra M, et al. (2007) Simile-Exit procedure for the management of gastroschisis. IberoAmerican Journal of Fetal and Perinatal Medicine 1(1): 7-12.

18. Galdon I, Rojas E, Hernández A, Alejandro José (2014) Simil Exit versus Primary Closure of the abdominal wall in newborns with gastroschisis. Arch Ven Puer and Ped 77(2): 65-70.

19. Plaza F, Espinoza L, Colmenares G, Plaza F Uses of the placenta and the umbilical cord. Gac Med Car.

20. Heaton FC, Thomas C, Owen J (1970) The use of umbilical cord for reconstruction of abdominal wall defects. Surg Forum 21: 56-57.

21. Samii AM, Jafroudi Y (1974) Gastroschisis: use of umbilical cord in its repair.J Med Liban 27(4): 473-477.

22. Zivković S (1991) Repair of gastroschisis using umbilical cord as a patch. J Pediatr Surg 26(10): 1179-1180.

23. Werbeck R, Koltai J (2011) Umbilical cord as temporary coverage in gastroschisis. Eur J Pediatr Surg 21(5): 292-295.

24. Hernandez S, Lopez T, Fernandez E, Trojan M, Luque J (2007) Gastroschisis: primary closure using umbilical cord strengthened by a polypropylene mesh. J Perinat Med 35(3): 249-251.

25. Ionescu S, Andrei B, Tirlea S, Bunea B, Licsandru E, Cistoveanu C et al. (2013) Considerations on gastroschisis repair. Chirugia (Bucur) 108(4): 509-515.

26. Ejike C, Mathur M (2012) Abdominal Decompression in Children. Crit Care Res and Pract.

27. Carnaghan H, Pereira S, James C, Charlesworth P, Ghionzoli M, et al (2014) Is early delivery beneficial in gastroschisis? J Pediatr Surg 49(6): 928-933.

28. Calcagnotto H, Müller A, Leite J, Sanseverino M, Gomes K, et al. (2013) Factors associated with mortality in recém-nascidos com gastrosquise. Rev Bras Ginecol Obstet 35(12): 549-553.

29. Tarca E, Aprodu S (2013) Gastroschisis treatment: what are the causes of high morbidity and mortality rates? Chirurgia (Bucur) 108(4): 516-520.

30. Capecchi G, Count A, Rovere L, Sasia F, Oxilia H, et al. (2008) Congenital defects of the abdominal wall. Most common pathologies: gastroschisis and omphalocele. Anu Fund Dr. J. R. Villavicencio 16: 45-50.

31. Shao L, Hou G, Zhang E (2006) Appraisal of the repair gastroschisis with autogenous umbilical cord. Zhonghua Wai Ke Za Zhi 44(21): 1490-1492.

32. Ross AR, Eaton S, Zani A, Ade-Ajayi N, Pierro A, et al. (2015) The role of preformed silos in the management of infants with gastroschisis: a systematic review and meta-analysis. Pediatr Surg Int 31(5):473-83.

33. Wright N, Zani A, Ade-Ajayi N (2015) Epidemiology, management and outcome of gastroschisis in Sub-Saharan Africa: Results of an international survey. Afr J Paediatr Surg 12(1): 1-6.

34. Allin B, Tse W, Marven S, Johnson P, Knight M. (2015) Challenges of improving the evidence base in smaller surgical specialties, as highlighted by a systematic review of Gastroschisis Management. PLOS ONE 10(1): e0116908. 
RPN Research in Pediatrics \&
Neonatology ser $\rightarrow \frac{15}{2}$

High-level peer review and editorial services

- Freely accessible online immediately upon publication

- Authors retain the copyright to their work

- Licensing it under a Creative Commons license

- Visibility through different online platforms 